\title{
Comparing Health-related Quality of Life of Lenke 1-2, and Lenke 5-6 Severe AIS Adolescent Idiopathic Scoliosis Patients 2 Years after Surgery Using SRS-22 Questionnaire
}

\author{
Wai-Wang Chau ${ }^{1}$, Victor Illescas ${ }^{2}$, Bobby Kin-Wah $\mathrm{Ng}^{3}$
}

\begin{abstract}
Introduction: Lenke classification organizes curve patterns into six major "curve types". Health-related quality of life (HRQOL) in AIS patients is gaining attention particularly on whose undergone surgery and findings are sometimes not expected. Comparisons of HRQOL in severe patients between Lenke 1 (main thoracic) and Lenke 2 (double thoracic) and patients with Lenke 5 (thoracolumbar/lumbar) and Lenke 6 (thoracolumbar/ lumbar-main thoracic) curve types have yet to be carried out.

Materials and methods: Forty-six severe AIS patients classified Lenke type 1, 2, 4, and 5 undergone surgery from 2016 to 2019 were recruited. Demographic variables and surgical details were collected. Patients filled out the SRS-22 questionnaire at (1) Before surgery (preoperative), (2) Before hospital discharge (post-op1), (3) 1 year postoperative (post-op2), and (4) 2 years post-op (post-op3). Statistical comparisons of HRQOL domain scores were carried out between Lenke $1+2$ group and Lenke $5+6$ group longitudinally and between groups.

Results: The mean age at surgery is 18.14 years. Longitudinal comparisons showed "Function" and "Pain" scores dropped before hospital discharge and recovered at post-op follow-ups. "Function" and "Pain" in Lenke $1+2$ group at between-group comparisons were significantly higher than Lenke $5+6$. Self-image, satisfaction, and mean scores were also higher in Lenke $1+2$ without statistical significance.

Conclusion: All domains showed improvements 2 years after surgery in both Lenke type groups, of which significant improvements were statistically found in "Function", "Pain", and "Mental health" in Lenke $1+2$ patients.

Key messages:

- All HRQOL domains in both Lenke $1+2$ (main thoracic + double thoracic) group and Lenke $5+6$ (thoracolumbar/lumbar + thoracolumbar/ lumbar-main thoracic) group improved after spinal surgery.

- "Function","Pain", and“Mental health"in patients of Lenke $1+2$ group significantly were improved statistically than patients in Lenke $5+6$ group.

- "Self-image" and "Satisfaction" were also improved without statistical significance.

Keywords: Adolescent idiopathic scoliosis, Lenke classification, Quality of life, Surgery.

Journal of Orthopedics and Joint Surgery (2021): 10.5005/jp-journals-10079-1039
\end{abstract}

\section{INTRODUCTION}

Lenke classification established in 2001 being a new classification system with three components: curve type, a lumbar spine modifier, and a sagittal thoracic modifier. ${ }^{1,2}$ Apart from a few reports on surgical treatment of Lenke 1 (main thoracic) and Lenke 2 (double thoracic) suffering severe AIS patients, and similar reports on Lenke 5 (thoracolumbar/lumbar) and Lenke 6 (thoracolumbar/lumbarmain thoracic) severely suffering AIS patients. Surprisingly, none discussed the health-related quality of life (HRQOL) of these two groups of patients. Structural changes after surgery leading to change in the quality of life in these two groups of patients have yet to be discussed. Surgeons and researchers might have their underlying "answers" to the changes of HRQOL after surgery in these two groups of patients, however, no study has been carried out to prove their "answers" and the results may be different from expectations.

Surgical strategy on the effectiveness of fusion approach [applying anterior (ASF) or posterior (PSF) or both (ASF + PSF)] and levels was widely discussed. O'Donnell et al. reported ASF had saved a fusion level but required more surgical time than PSF. $^{3}$ Another study demonstrated anterior approach provided greater correction of thoracolumbar curves when treating Lenke 5 c curves. ${ }^{4}$ Major thoracolumbar/lumbar curve fusion in Lenke 5C $\overline{{ }^{1-3} \text { Department of Orthopaedics and Traumatology, Chinese University }}$ of Hong Kong, Shatin, NT, Hong Kong

Corresponding Author: Bobby Kin-Wah Ng, Department of Orthopaedics and Traumatology, Chinese University of Hong Kong, Shatin, NT, Hong Kong, Phone: +(852) 9680-0776, e-mail: bobng@ort. cuhk.edu.hk

How to cite this article: Chau WW, Illescas V, Ng BKW. Comparing Health-related Quality of Life of Lenke 1-2, and Lenke 5-6 Severe AIS Adolescent Idiopathic Scoliosis Patients 2 Years after Surgery Using SRS-22 Questionnaire. J Orth Joint Surg 2021;3(1): 23-30.

Source of support: Nil

Conflict of interest: None

AIS could be achieved by both anterior and posterior techniques. ${ }^{5} \mathrm{~A}$ Japanese group included the SRS-22 questionnaire in the evaluation of posterior correction and fusion surgery using pedicle-screw constructs for Lenke type 5 C AIS patients. ${ }^{6}$ However, results on SRS-22 were debatable without showing any actual data suggesting the quality of life assessment was only a minor part of this study. ${ }^{6}$ Structural differences in these types of patients were definitive; however, the HRQOL of these two types of patients who operated 
has not been discussed. Majority of the articles discussing Lenke 1 $+2^{7,8}$ and Lenke $5+6^{3,9-11}$ patients focused on surgical techniques and approaches leading to promising surgical outcomes in terms of cosmetic improvements and truck balance.

Adolescent idiopathic scoliosis (AIS) is a three-dimensional deformity presenting with back deformity, a rib hump, and/or shoulder asymmetry. ${ }^{12}$ The etiology is complex and still unknown although the genetic influence is reported to have played a major role. ${ }^{13-18}$ The prognosis of AIS depends on several factors including curve morphology, trunk imbalance, curve magnitude, age of onset, stage of bone growth, and rate of progression. ${ }^{13-15,19}$ Progression results in body deformities such as uneven shoulders, and asymmetric waistline that leads to chronic pain, decreased or poor function self-image and mental health, and in severe cases disturbed pulmonary function. ${ }^{20-23}$ There is a higher risk of health problems beyond maturity once the Cobb angle exceeds $50^{\circ}$ in a progressive curve, causing cosmetic deformity and progressive functional limitations. ${ }^{13,14,20-23}$ The current indications of surgery are curves $>45-50^{\circ}$ at skeletal maturity or patients with $1-2$ years of growth remaining..$^{15,19}$ Our team recently published a report on the changes in HRQOL in operative AIS patients over 30 years, and the patients were at their perimenopause. ${ }^{24}$

The outcome of corrective scoliosis surgery is dependent on radiographic improvements as well as the patient's perspective and satisfaction..$^{25}$ Objective success in correction is not correlated with subjective satisfaction of the patient and the family because their perception of appearance differs from that of surgeons and other factors. ${ }^{26}$ Improvements in the general self-image, function, level of activity domains, and pain are reported after surgical correction and had no significant correlation between the magnitude of curve correction and outcome scores. ${ }^{13,27}$ SRS-22 is proven to be a valid and reliable instrument for assessing patients with AIS that is sensitive to changes following surgery. ${ }^{28}$ The questionnaire is reliable with internal consistency and reproducibility. It is shorter and more focused on the health issues related to idiopathic scoliosis than SF-36. ${ }^{29}$

Literatures on the quality of life of AIS patients who have undergone surgery were few. The underestimation of different HRQOL scores on major complications in lumbar degenerative scoliosis surgery in 138 patients was an important finding, although the data generalizability was limited to adult patients (mean age: 59.8) and those who underwent lumbar deformities. ${ }^{30}$ Another single-institute study on the effect of age on the quality of life for adult patients with spinal deformity after surgery demonstrated age was an important factor in patients' perceptions of their functions and mental health. ${ }^{31}$ Another Japanese group worked on long-term health outcomes in severe AIS patients and noticed significant decreases in function and self-image with controls many years after surgery. ${ }^{32,33}$ The statistical comparisons were straightforward without considering any preoperative conditions. A literature review on the influences of surgery on the quality of life of AIS patients using SRS outcomes concluded general improvements in pain and self-image domains from baseline to 2 years postoperatively, and the natural history of AIS was important in surgical decision-making. ${ }^{34}$ Health-related quality of life in AIS shoulder balance in Lenke 1 and 2 curve types as well as curve flexibility on patient-centric outcomes were published. ${ }^{35,36}$ However, the lack of discussion on the relationship between the change in spinal curvatures before and after surgery and HRQOL explained by SRS domains remains an important area to explore.
The purpose of this study is to compare the HRQOL of surgical AIS patients between curve type Lenke $1+2$ and Lenke $5+6$ using SRS-22.

\section{Materials and Methods}

We reviewed 46 AIS patients who had undergone spinal surgery from 2016 to 2019. Informed consent was signed and obtained from every study participant and their legal guardians as required. Ethical approval was obtained from the ethics review board of the joint NTEC/CUHK Ethics Committee (Research Ethics Committee approval number: 2019.213).

Demographic variables, e.g., age at surgery, years of follow-up, sex, Lenke stage, fusion levels, number of fusion levels, surgical and clinical details, and measurements were collected.

\section{SRS-22 Questionnaire}

SRS-22 protocol and methods to deliver the questionnaire through mobile devices have been discussed in our previous report. ${ }^{24}$ Briefly speaking, severe AIS patients completed the SRS-22 questionnaire before surgery (preoperative), immediate before hospital discharge after surgery (post-op 1), 1 year postoperatively (post-op 2), and 2 years postoperatively through mobile devices.

\section{Statistical Analysis}

Demographic variables of both groups were calculated and compared using Student's T-test (mean \pm standard deviation) or Chi-square test $[N(\%)]$ where appropriate. Surgical information regarding the three curvatures was tabulated. SRS-22 domain scores (function, pain, self-image, mental, satisfaction, and mean) and before surgery (preoperative) and follow-up sessions after surgery, i.e., immediate before hospital discharge (post-op 1), 1 year after surgery (post-op 2), and 2 years after surgery (post-op 3) were compared using one-way ANOVA. Other comparisons of SRS-22 domain scores using follow-up period instead of follow-up sessions ( $\leq 12,12-24$, and $>24$ months) were also carried out using one-way ANOVA. Between-group comparisons (between Lenke $1+2$ group vs Lenke $5+6$ group) at different time points were performed using Student's $T$-test. Data analysis was carried out using IBM SPSS 26.0 (Armonk, New York). A two-sided $p$ value $\leq 0.05$ was considered statistically significant.

\section{Results}

The mean age at surgery is 18.14 years (Table 1 ). The average number of pedicle screws placed was 17.75 with an average pedicle screw density of $73.49 \%$. The mean years of follow-up were 1.53 years (range: $0.25-2.75$ years).

\section{Surgical Information}

Statistical significances were observed in most of the main thoracic curves and all lumbar curve characteristics comparing between the two groups (Table 2).

Table 1A: Demographic variables (numeric) (mean \pm SD)

\begin{tabular}{lccl}
\hline $\begin{array}{l}\text { Demographic } \\
\text { variable }\end{array}$ & $\begin{array}{l}\text { Lenke } 1 \text { and } 2 \\
(N=26)\end{array}$ & $\begin{array}{l}\text { Lenke 4 and } 5 \\
(N=20)\end{array}$ & p value \\
\hline Age & $18.0 \pm 3.9$ & $18.3 \pm 4.5$ & 0.82 \\
Years of follow-up & $1.5 \pm 0.8$ & $1.6 \pm 0.7$ & 0.85 \\
\hline
\end{tabular}




\section{SRS-22 Domain Scores across the Treatment Periods}

SRS-22 mean scores were calculated through two different approaches on how the follow-up period was classified (i.e., $X$ axis) (Table 3). The follow-up period was classified by (1) follow-up time points (preoperative, postoperative 1, postoperative 2, and postoperative 3 ), and ( 2$) \leq 12,12-24$, and $>24$ months since the surgery. The reason for using these two classifications serves two purposes: (1) the first series showed the SRS-22 domain scores by clinic visit without considering the period between follow-up, and

Table 1B: Demographic variables (categorical) [N (\%)]

\begin{tabular}{lccc}
\hline $\begin{array}{l}\text { Demographic } \\
\text { variable }\end{array}$ & $\begin{array}{l}\text { Lenke } 1 \text { and } 2 \\
(N=26)\end{array}$ & $\begin{array}{l}\text { Lenke } 4 \text { and } 5 \\
(N=20)\end{array}$ & p value \\
\hline Sex & & & \\
Male & $6(23.1)$ & $5(25.0)$ & 0.58 \\
Female & $20(76.9)$ & $15(75.0)$ & \\
Lenke stage & & & - \\
1 & $9(34.6)$ & - & \\
2 & $17(65.4)$ & - & \\
4 & - & $10(50.0)$ & \\
5 & - & $10(50.0)$ & \\
Fusion levels & & & \\
From T2 & $13(50.0)$ & $2(10.0)$ & $<0.01$ \\
From T3 & $7(26.9)$ & $4(20.0)$ & \\
From T4 & $6(23.1)$ & $3(15.0)$ & \\
From T5 & 0 & $2(10.0)$ & \\
From T9 & 0 & $5(25.0)$ & \\
From T10 & 0 & $3(15.0)$ & \\
From T11 & 0 & $1(5.0)$ & \\
Number of fused levels & & \\
$\leq 10$ & $11(42.3)$ & $11(55.0)$ & 0.31 \\
11 & $5(19.2)$ & $2(10.0)$ & \\
12 & $4(15.4)$ & $6(30.0)$ & \\
13 & $4(15.4)$ & $1(5.0)$ & \\
14 & $2(7.7)$ & 0 & \\
\hline
\end{tabular}

(2) the second series defined the $x$-axis as a definitive follow-up period which made the comparisons more clinically meaningful.

The corresponding findings were also graphically presented in two figure series (Figs 1 and 2). "Function" and "Pain" scores showed a similar pattern of score changing over the four time points. Mean scores were dropped at "Post-op 1" and gradually returned back to "Preoperative 1". The mean scores at "Post-op 3" were higher than "Post-op 2"; however, the mean score differences between "Post-op 3" and "Post-op 2" were much smaller compared with the differences between "Post-op 1" and "Preoperative". The mean scores of "Mental", "Self-image", and "Satisfaction" were gradually increasing over the four time points, e.g., from 3.24, 3.74, 4.06, to 4.08 in "Self-image". "Mean" scores were momentously similar at the four time points. Except "Mental", statistical significances were observed in the other five mean scores.

The patterns of SRS-22 score changes over the three time periods were similar in the six domain scores except for a slight difference in "Satisfaction", which showed an increase 12-24 months after surgery, and the scores at " $>24$ months" returned to the scores.

In between-group comparisons, Lenke $1+2$ patients scored significantly higher than Lenke $5+6$ patients in "Function" at Post-op 2 (4.4 vs 3.9, $p=0.01$ ) and Post-op 3 (4.6 vs 3.9, $p=0.01$ ), as well as "Pain" (4.0 vs 3.1, $p<0.01$ ), "Mental" (4.1 vs 3.3, $p<0.01$ ), and "Mean" (3.9 vs 3.4, $p=0.01$ ) at Post-op 1. The significantly better "Function" domains in Lenke $1+2$ patients observed above (by follow-up sessions) was also observed when follow-up periods applied. "Function" in Lenke $1+2$ patients was significantly better than Lenke $5+6$ patients at $<12$ months ( 4.2 vs 3.8, $p=0.01$ ), and $12-24$ months (4.6 vs $3.9, p=0.03$ ) postoperatively.

\section{Discussion}

Comparing with AIS Lenke $5+6$ patients, AIS Lenke $1+2$ patients demonstrated significantly persistent improvements in their functional capabilities after spinal correction until 2 years postoperatively, better pain management, and mental health at hospital discharge after surgery. Self-image, Satisfaction, and Mean showed no difference between the two groups from before surgery to 2 years after surgery.

Table 2: Comparing surgical information and different Cobb angles between patients at Lenke 1 and 2, and patients at Lenke 5 and 6 (mean \pm SD)

\begin{tabular}{|c|c|c|c|}
\hline Surgical and clinical details and measurements & Lenke 1 and 2 & Lenke 5 and 6 & $p$ value \\
\hline \multicolumn{4}{|l|}{ Proximal thoracic curve } \\
\hline Proximal thoracic curve (preoperative Cobb) & $40.1 \pm 14.9$ & - & - \\
\hline Proximal thoracic curve (Left SB Cobb) & $33.5 \pm 14.8$ & - & - \\
\hline Proximal thoracic curve (postoperative Cobb) & $21.7 \pm 8.5$ & - & - \\
\hline Proximal thoracic curve (FU Cobb) & $23.7 \pm 10.3$ & - & - \\
\hline \multicolumn{4}{|l|}{ Main thoracic curve } \\
\hline Main thoracic curve (preoperative Cobb) & $66.3 \pm 12.1$ & $40.7 \pm 14.4$ & $<0.01$ \\
\hline Main thoracic curve (Right SB Cobb) & $42.7 \pm 13.6$ & $31.6 \pm 14.8$ & 0.01 \\
\hline Main thoracic curve (postoperative Cobb) & $17.4 \pm 6.6$ & $19.7 \pm 5.9$ & 0.22 \\
\hline Main thoracic curve (FU Cobb) & $18.7 \pm 7.1$ & $22.5 \pm 6.8$ & 0.08 \\
\hline \multicolumn{4}{|l|}{ Lumbar curve } \\
\hline Lumbar curve (preoperative Cobb) & $32.9 \pm 8.9$ & $62.8 \pm 8.1$ & $<0.01$ \\
\hline Lumbar curve (Left SB Cobb) & $8.0 \pm 6.7$ & $32.5 \pm 10.1$ & $<0.01$ \\
\hline Lumbar curve (postoperative Cobb) & $7.7 \pm 5.3$ & $18.9 \pm 8.3$ & $<0.01$ \\
\hline Lumbar curve (FU Cobb) & $8.7 \pm 7.5$ & $22.8 \pm 9.1$ & $<0.01$ \\
\hline
\end{tabular}

Notes: SB, side bending; FU, follow-up 
HRQOL in AIS Different Lenke Stages Contributors

Table 3A: SRS-22 domains scores comparing among preoperative and succeeding follow-up consultations

\begin{tabular}{|c|c|c|c|c|c|}
\hline SRS-22 domain scores & Preoperative & Post-op 1 & Post-op 2 & Post-op 3 & $p$ value \\
\hline \multicolumn{6}{|l|}{ Lenke 1 and 2} \\
\hline Years since & - & - & $1.0 \pm 0.7$ & $1.6 \pm 0.8$ & \\
\hline Function & $4.6 \pm 0.4^{\mathrm{a}}$ & $3.4 \pm 1.0^{\mathrm{a}, \mathrm{b}, \mathrm{c}}$ & $4.4 \pm 0.5^{b}$ & $4.6 \pm 0.4^{c}$ & $<0.01$ \\
\hline Pain & $4.3 \pm 0.6$ & $4.0 \pm 0.5^{\mathrm{d}, \mathrm{e}}$ & $4.4 \pm 0.6^{d}$ & $4.6 \pm 0.3^{e}$ & 0.11 \\
\hline Self-image & $3.2 \pm 0.4^{f, g, h}$ & $3.8 \pm 0.5^{f}$ & $4.0 \pm 0.5^{g}$ & $4.1 \pm 0.5^{h}$ & $<0.01$ \\
\hline Mental & $4.0 \pm 0.6$ & $4.1 \pm 0.6$ & $4.3 \pm 0.5$ & $4.1 \pm 0.7$ & 0.47 \\
\hline Satisfaction & $4.0 \pm 0.9$ & $4.0 \pm 0.8$ & $4.4 \pm 0.5$ & $4.4 \pm 0.6$ & 0.16 \\
\hline Mean & $4.0 \pm 0.3$ & $3.9 \pm 0.5$ & $4.30 \pm 0.4$ & $4.4 \pm 0.4$ & $<0.01$ \\
\hline \multicolumn{6}{|l|}{ Lenke 5 and 6} \\
\hline Years since & - & - & $0.8 \pm 0.9$ & $1.4 \pm 0.5$ & \\
\hline Function & $4.5 \pm 0.4^{i}$ & $3.2 \pm 0.8^{\mathrm{i}, j}$ & $3.9 \pm 0.5^{j}$ & $3.9 \pm 0.6$ & $<0.01$ \\
\hline Pain & $4.3 \pm 0.6^{k}$ & $3.1 \pm 0.9^{k, l, m}$ & $4.3 \pm 0.6^{1}$ & $4.0 \pm 0.8^{m}$ & $<0.01$ \\
\hline Self-image & $3.0 \pm 0.6^{n, o}$ & $3.6 \pm 0.4$ & $4.1 \pm 0.5^{n}$ & $3.9 \pm 0.6^{\circ}$ & $<0.01$ \\
\hline Mental & $3.7 \pm 0.6$ & $3.3 \pm 0.5$ & $3.9 \pm 0.6$ & $3.6 \pm 0.7$ & 0.10 \\
\hline Satisfaction & $4.1 \pm 1.0$ & $4.0 \pm 0.8$ & $4.6 \pm 0.5$ & $4.3 \pm 0.8$ & 0.25 \\
\hline Mean & $3.9 \pm 0.4^{p}$ & $3.4 \pm 0.4^{p, q}$ & $4.2 \pm 0.4^{q}$ & $3.9 \pm 0.6$ & $<0.01$ \\
\hline
\end{tabular}

a to q: statistical significance pairs at $p<0.05$

Table 3B: SRS-22 domains scores comparing among follow-up time points

\begin{tabular}{|c|c|c|c|c|}
\hline SRS-22 domain scores & $\leq 12$ months & 12-24 months & $>24$ months & $p$ value \\
\hline \multicolumn{5}{|l|}{ Lenke 1 and 2} \\
\hline Months since surgery & $6.6 \pm 3.6$ & $20.4 \pm 3.1$ & $30.8 \pm 2.9$ & - \\
\hline Function & $4.3 \pm 0.4^{\mathrm{a}}$ & $4.6 \pm 0.4$ & $4.9 \pm 0.2^{\mathrm{a}}$ & 0.02 \\
\hline Pain & $4.3 \pm 0.6$ & $4.5 \pm 0.5$ & $4.9 \pm 0.2$ & 0.23 \\
\hline Self-image & $4.0 \pm 0.5$ & $4.1 \pm 0.5$ & $4.6 \pm 0.4$ & 0.11 \\
\hline Mental & $4.0 \pm 0.5$ & $4.4 \pm 0.5$ & $4.7 \pm 0.4$ & 0.04 \\
\hline Satisfaction & $4.4 \pm 0.6$ & $4.4 \pm 0.5$ & $4.6 \pm 0.5$ & 0.78 \\
\hline Mean & $4.2 \pm 0.4$ & $4.4 \pm 0.4$ & $4.7 \pm 0.2$ & 0.06 \\
\hline \multicolumn{5}{|l|}{ Lenke 5 and 6} \\
\hline Months since surgery & $6.8 \pm 3.8$ & $19.2 \pm 3.3$ & $34.5 \pm 6.4$ & - \\
\hline Function & $3.8 \pm 0.4$ & $3.9 \pm 0.8$ & $4.6 \pm 0.3$ & 0.15 \\
\hline Pain & $4.3 \pm 0.5$ & $3.9 \pm 1.1$ & $4.3 \pm 1.0$ & 0.56 \\
\hline Self-image & $4.2 \pm 0.5$ & $3.8 \pm 0.6$ & $3.9 \pm 0.4$ & 0.38 \\
\hline Mental & $3.8 \pm 0.5$ & $3.6 \pm 1.0$ & $4.3 \pm 1.0$ & 0.48 \\
\hline Satisfaction & $4.6 \pm 0.5$ & $3.9 \pm 0.8$ & $5.0 \pm 0.0$ & 0.05 \\
\hline Mean & $4.1 \pm 3.4$ & $3.8 \pm 0.8$ & $4.4 \pm 0.5$ & 0.28 \\
\hline
\end{tabular}

a: statistical significance pair at $p<0.05$

Surprisingly, "Self-image" and "Satisfaction" (Satisfaction with management) were similar between Lenke $1+2$ and Lenke $5+6$ patients throughout the study period (before and after 2 years of surgery). Self-image was kept improving after spinal correction in both groups. This a novel finding in which most of the surgeons and clinicians expected self-image was much improved in Lenke $5+6$ patients than Lenke $1+2$ in terms of different regional structural characteristics. Correcting the major curvatures at main thoracic (MT) or double thoracic (DT) provide a similar effect when correcting at thoracolumbar/lumbar (TL/L) or thoracolumbar/lumbar-MT (TL/L-MT). Our published article showed female with Lenke 2 curve before surgery was the group which was best beneficial from the surgery using regression models. ${ }^{36}$ At that time, however, we did not consider the effects of different Lenke curvatures on HRQOL. Through this study results, self-image improves after surgery regardless of curve types and patients. Therefore, the regional structural characteristics (curve type) are not crucial factors when considering surgery. In addition, results from our another article concluded better self-image could be conserved when surgery was performed on patients with higher curve flexibility. ${ }^{35}$ As a result, curve flexibility, not curve type, is a factor to consider surgery.

Function, pain management, and mental health improved significantly in AIS Lenke $1+2$ patients than Lenke $5+6$ patients. Lenke 1 patients showed that distal segments were more flexible than the proximal ones as seen through the differences in fulcrum-bending flexibility. ${ }^{37}$ A Spanish group reviewing articles concerning AIS bracing and surgical patients and found patients treated surgically were found to have a better self-image than when they were on the brace. ${ }^{38}$ Our reports showed better self-image after an operation and that could be associated with better curve 

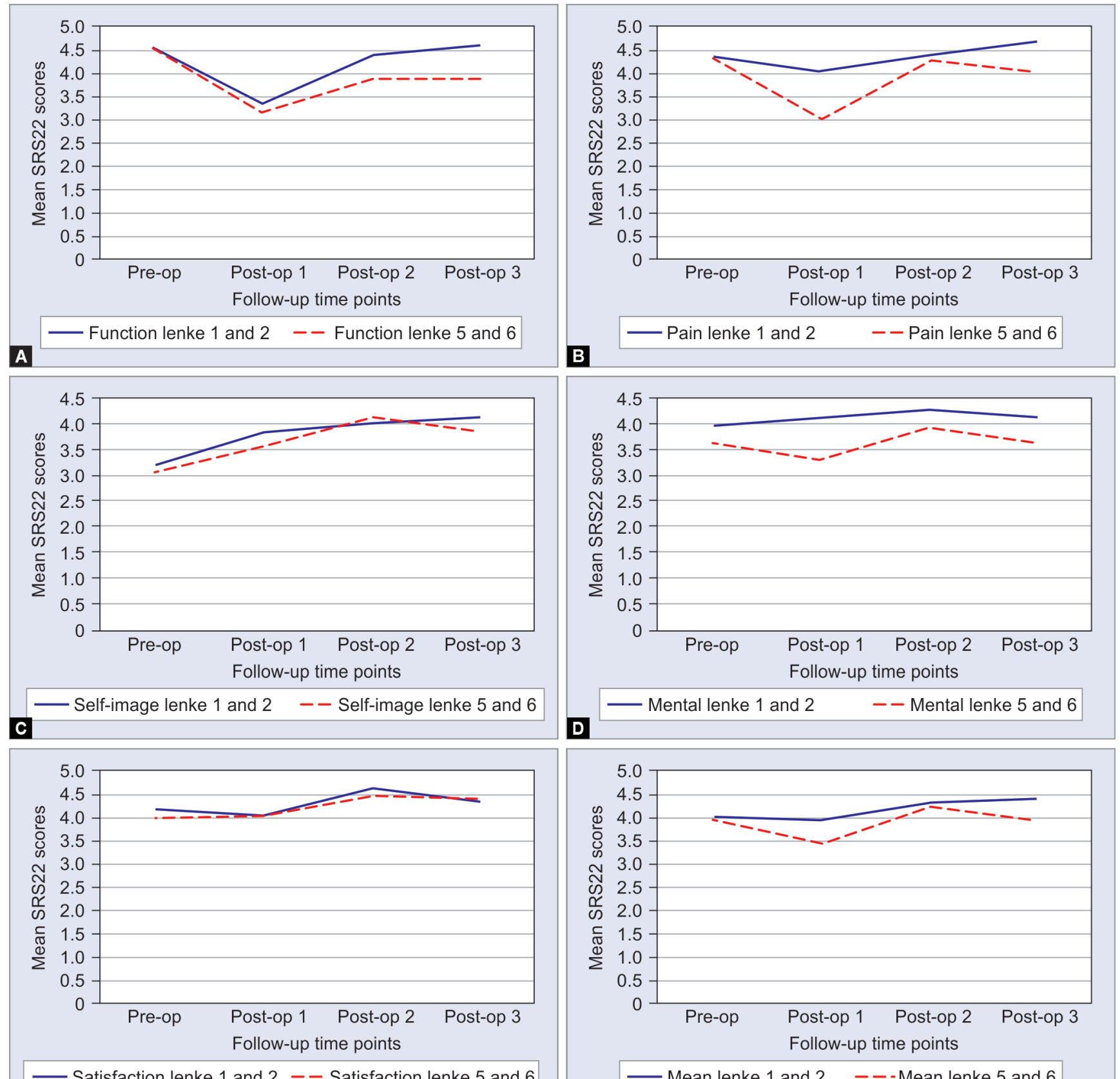

$\mathbf{E}$

Satisfaction lenke 1 and 2 - Satisfaction lenke 5 and 6

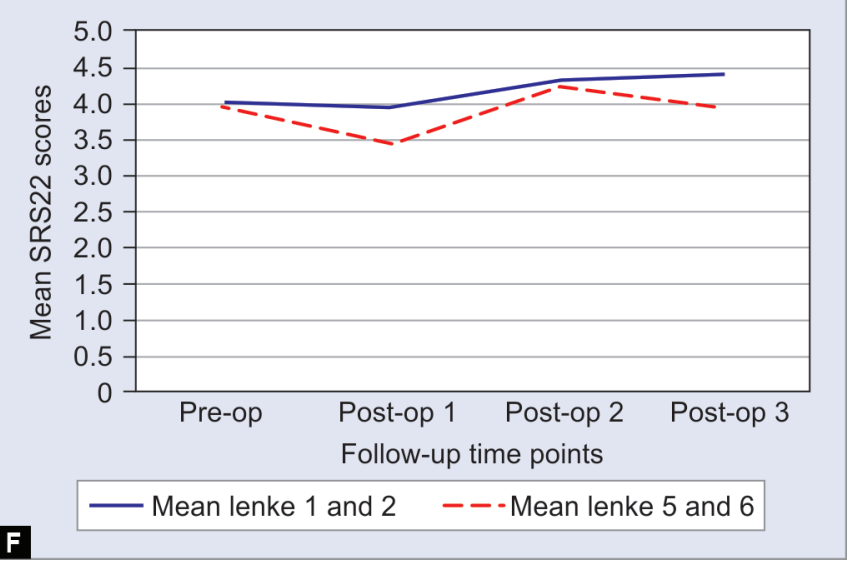

Figs 1 A to F: (A) SRS22 function scores of Lenke 1 and 2, and Lenke 5 and 6 patients during follow-up time points; (B) SRS22 pain scores of Lenke 1 and 2, and Lenke 5 and 6 patients during follow-up time points; (C) SRS22 self-image scores of Lenke 1 and 2, and Lenke 5 and 6 patients during follow-up time points; (D) SRS22 mental scores of Lenke 1 and 2, and Lenke 5 and 6 patients during follow-up time points; (E) SRS22 satisfaction scores of Lenke 1 and 2, and Lenke 5 and 6 patients during follow-up time points; (F) SRS22 mean scores of Lenke 1 and 2, and Lenke 5 and 6 patients during follow-up time points

flexibility. ${ }^{36}$ Function and pain were dropped immediately after surgery and recovered back to pre-surgical level, and mental health was relatively stable. ${ }^{39}$ Searching for eligible studies on HRQOL in severe AIS patients with TL/L and/or TL/L-MT curve types returned very few results. A European group reported the radiological and clinical outcomes of 78 AIS Lenke 5 patients who underwent either selective (35) or hyperselective (43) posterior fusions with a minimum of 2 years postoperative follow-up. ${ }^{40}$ SRS-2 2 function domain score was significantly better after short-segment fusions $(p=0.03)$ and SRS-22 pain scores were above 4 in both groups. ${ }^{40}$
A Colorado-based AIS study on Lenke 5 curve types treated with either anterior $(N=98)$ or posterior spinal fusion $(N=51)$ measuring their SRS-22 outcomes found no statistically significant difference between the ASF and PSF groups both preoperatively and at 2 years $(p>0.05) .^{3}$ This study summarized and compared the HRQOL of Lenke $1+2$ as well as Lenke $5+6$ AIS surgical subjects. This article compared the HRQOL of two groups of severely suffering AIS patients with relatively different curve types.

Discussions on the relationship between Lenke curve types and HRQOL were scarce. A French-based study of 45 patients 
HRQOL in AIS Different Lenke Stages Contributors
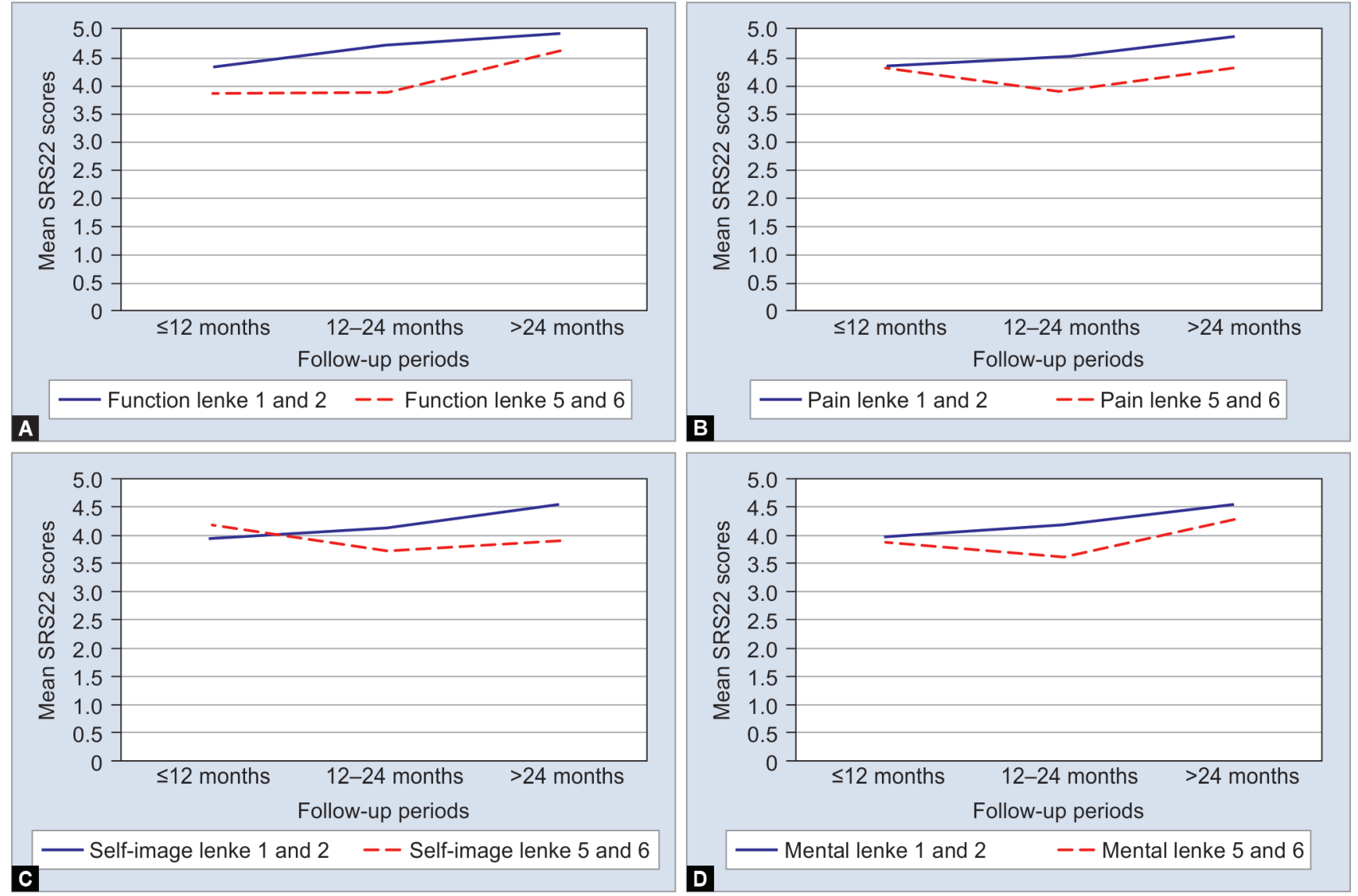

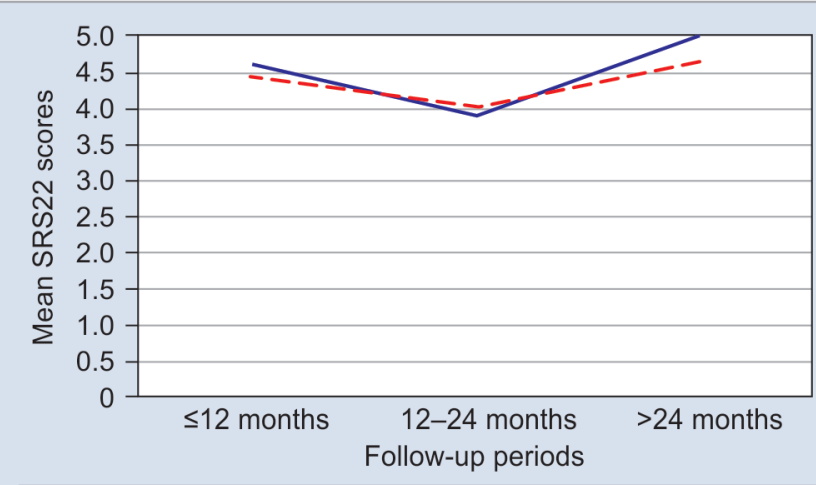

- - Satisfaction lenke 1 and 2 - Satisfaction lenke 5 and 6

\section{百}

Figs $2 A$ to F: (A) SRS22 function scores of Lenke 1 and 2, and Lenke 5 and 6 patients $\leq 12$ months, $12-24$ months, and $>24$ months after surgery; (B) SRS22 pain scores of Lenke 1 and 2, and Lenke 5 and 6 patients $\leq 12$ months, $12-24$ months, and > 24 months after surgery; (C) SRS22 Self-image scores of Lenke 1 and 2, and Lenke 5 and 6 patients $\leq 12$ months, 12-24 months, and >24 months after surgery; (D) SRS22 Mental scores of Lenke 1 and 2, and Lenke 5 and 6 patients $\leq 12$ months, 12-24 months, and >24 months after surgery; (E) SRS22 Satisfaction scores of Lenke 1 and 2 , and Lenke 5 and 6 patients $\leq 12$ months, 12-24 months, and $>24$ months after surgery; (F) SRS22 mean scores of Lenke 1 and 2, and Lenke 5 and 6 patients $\leq 12$ months, $12-24$ months, and $>24$ months after surgery

(Lenke $1=21$, Lenke $2=2$, Lenke $3=13$, Lenke $5=9$ ) fully completed SRS-22 at their 2 years or more after surgery, and results showed no difference in functional SRS-22 health status in patients according to Lenke classification. ${ }^{41}$ However, the results were poorly convinced because the authors employed ANOVA as the statistical test by comparing domain scores with different Lenke types provided very few sample sizes in certain Lenke types. A retrospective review of patient and spinal curve characteristics in 1,912 surgical cases with Lenke types showed Lenke type 4 patients had lower preoperative SRS self-image scores than patients with type 1 curves $(p=0.01)$. We observed no difference in preoperative self-image scores between Lenke type $5+6$ and 1 $+2 .{ }^{42}$ Lenke type 4 donates triple major with structural proximal thoracic curvature, while type 5 and 6 represent non-structural proximal thoracic curves. Lenke type 1 curve also denotes nonstructural proximal thoracic curvatures and that would explain the discrepancy. A Japanese research group looked for factors contributing to self-image (SRS-22r) in AIS. ${ }^{43}$ One example with 
Lenke type 1AN scored the SRS-22r self-image before and after 2 years of surgery as 1.8 and 4.8 , respectively. Another example of Lenke type $1 \mathrm{C}$ scored 3.0 and 3.2, respectively. The authors concluded apical vertebral translation at preoperative and persistent thoracolumbar/lumbar curvature were associated with a low self-image. The authors did not go into details utilizing the SRS-22 data. This article provides extensive comparisons and reviews of HRQOL in two different curve types.

Ceiling effect in SRS-22 self-image and mental domains is a known documented criticism of SRS questionnaires ${ }^{44-46}$ and in subcategories. ${ }^{47}$ Caronni et al. created the SRS-7 despite sacrificing the ability to detect HRQOL. ${ }^{45} \mathrm{~A}$ recent systematic review and meta-analysis mentioned the ceiling effect when assessing the functional changes would become less sensitive in those highly functional patients. ${ }^{48}$ SRS-22 function domains in both groups are above 4 before surgery, therefore, we believe the ceiling effect in this study is not detrimental to the results and findings in this study.

Further study is warranted by applying a similar research protocol on AIS patients with other Lenke curve types with increased sample sizes. Gender difference in the outcomes is another interesting topic. Moreover, the effect of different surgical approaches (anterior, posterior, or anterio-posterior) on severe AIS Lenke $1+2$ and $5+6$ would worth exploring the influences of mechanical changes in spinal geometry on these two types of patients and their health-related outcomes.

\section{Limitations}

The statistical results might have been limited by the number of patients in both groups. Recall bias being the retrospective nature of this study is inevitable. Reactions to surgical intervention are individual and that affected very much on perception of healthrelated outcomes. The ceiling effect in SRS-22 self-image and mental domains is a known issue particularly for postoperative patients, however, the effect is not significant in this study possibly controlled by Lenke types.

\section{Conclusion}

Spinal correction in AIS patients improves function, pain, and mental health, and the effects are significantly sorted in severe AIS Lenke $1+2$ (main thoracic + double thoracic) patients than Lenke $5+6$ (thoracolumbar/lumbar + thoracolumbar/lumbar-main thoracic). Self-image and Satisfaction were also improved without statistical significance after surgery in both groups.

\section{References}

1. Lenke LG, Betz RR, Harms J, et al. Adolescent idiopathic scoliosis: a new classification to determine extent of spinal arthrodesis. J Bone Joint Surg Am 2001;83(8):1169-1181. DOI: 10.2106/00004623-20010800000006.

2. Lenke LG. Lenke classification system of adolescent idiopathic scoliosis: treatment recommendations. Instr Course Lect 2005;54:537542.

3. O'Donnell C, Michael N, Pan X, et al. Anterior spinal fusion and posterior spinal fusion both effectively treat Lenke type 5 curves in adolescent idiopathic scoliosis: a multicenter study*. Spine Deformity 2018;6(3):231-240. DOI: 10.1016/j.jspd.2017.09.054.

4. Abel MF, Singla A, Feger MA, et al. Surgical treatment of Lenke 5 adolescent idiopathic scoliosis: comparison of anterior vs posterior approach. World J Orthop 2016;7(9):553-560. DOI: 10.5312/wjo. v7.i9.553.
5. Dong $Y$, Weng $X$, Zhao H, et al. Lenke 5 C curves in adolescent idiopathic scoliosis: anterior vs posterior selective fusion. Neurosurgery 2016;78(3):324-331. DOI: 10.1227/NEU.0000000000001055.

6. Okada E, Watanabe K, Pang L, et al. Posterior correction and fusion surgery using pedicle-screw constructs for Lenke type $5 \mathrm{C}$ adolescent idiopathic scoliosis: a preliminary report. Spine (Phila Pa 1976) 2015;40(1):25-30. DOI: 10.1097/BRS.0000000000000652.

7. Newton PO, Marks MC, Bastrom TP, et al. Surgical treatment of Lenke 1 main thoracic idiopathic scoliosis: results of a prospective, multicenter study. Spine (Phila Pa 1976) 2013;38(4):328-338. DOI: 10.1097/BRS.0b013e31826c6df4.

8. Albayrak A, Buyuk AF, Ucpunar $\mathrm{H}$, et al. Pre- and postoperative photographs and surgical outcomes in patients with Lenke type 1 adolescent idiopathic scoliosis. Spine (Phila Pa 1976) 2015;40(7):469474. DOI: 10.1097/BRS.0000000000000796.

9. Senkoylu A, Luk KDK, Wong YW, et al. Prognosis of spontaneous thoracic curve correction after the selective anterior fusion of thoracolumbar/lumbar (Lenke 5C) curves in idiopathic scoliosis. Spine J 2014;14(7):1117-1124. DOI: 10.1016/j.spinee.2013.07.467.

10. Luo $M$, Wang W, Shen $M$, et al. Anterior versus posterior approach in Lenke 5C adolescent idiopathic scoliosis: a meta-analysis of fusion segments and radiological outcomes. J Orthop Surg Res 2016;11(1):77. DOI: 10.1186/s13018-016-0415-9.

11. Chen J, Yang C, Ran B, et al. Correction of Lenke 5 adolescent idiopathic scoliosis using pedicle screw instrumentation: does implant density influence the correction? Spine (Phila Pa 1976) 2013;38(15):E946-E951. DOI: 10.1097/BRS.0b013e318297bfd4.

12. Cheng JC, Castelein RM, Chu WC, et al. Adolescent idiopathic scoliosis. Nat Rev Dis Prim 2015;1(1):15030. DOI: 10.1038/nrdp.2015.30.

13. Ghandehari H, Mahabadi MA, Mahdavi SM, et al. Evaluation of atient outcome and satisfaction after surgical treatment of adolescent idiopathic scoliosis using scoliosis research society-30. Archi Bone Joint Surg 2015;3:109-113.

14. Negrini S, Aulisa AG, Aulisa L, et al. 2011 SOSORT guidelines: orthopaedic and rehabilitation treatment of idiopathic scoliosis during growth. Scoliosis 2012;7(1):3. DOI: 10.1186/1748-7161-7-3.

15. Jr Z, Ma G. Adolescent idiopathic scoliosis: an in depth analysis and historical review. MOJ Orthop Rheumatol 2015. 3.

16. Dayer R, Haumont T, Belaieff W, et al. Idiopathic scoliosis: etiological concepts and hypotheses. J Child Orthop 2013;7(1):11-16. DOI: 10.1007/s11832-012-0458-3.

17. Forsberg $D$, Lundström C, Andersson M, et al. Model-based registration for assessment of spinal deformities in idiopathic scoliosis. Phys Med Biol 2014;59(2):311-326. DOI: 10.1088/00319155/59/2/311.

18. Weinstein SL, Dolan LA, Cheng JC, et al. Adolescent idiopathic scoliosis. Lancet 2008;371(9623):1527-1537. DOI: 10.1016/S01406736(08)60658-3.

19. Maruyama T, Takeshita K. Surgical treatment of scoliosis: a review of techniques currently applied. Scoliosis 2008;3(1):6. DOI: 10.1186/17487161-3-6.

20. Weinstein SL, Zavala DC, Ponseti IV. Idiopathic scoliosis: long-term follow-up and prognosis in untreated patients. J Bone Joint Surg Am 1981;63(5):702-712. DOI: 10.2106/00004623-198163050-00003.

21. Weinstein SL, Dolan LA, Spratt KF, et al. Health and function of patients with untreated idiopathic scoliosis: a 50-year natural history study. JAMA 2003;289(5):559-567. DOI: 10.1001/jama.289.5.559.

22. Collis DK, Ponseti IV. Long-term follow-up of patients with idiopathic scoliosis not treated surgically. J Bone Joint Surg Am 1969;51(3):425445. DOI: 10.2106/00004623-196951030-00001.

23. Weiss HR, Karavidas N, Moramarco M, et al. Long-term effects of untreated adolescent idiopathic scoliosis: a review of the literature. Asian Spine J 2016;10(6):1163-1169. DOI: 10.4184/asj.2016.10. 6.1163.

24. Chau WW, Ng BK, Hung AL. Health-related quality of life (HRQOL) of adolescent idiopathic scoliosis (AIS) patients from surgery to after 30 years using SRS-22 questionnaire. Spine Deform 2020;8(5):951-956. DOI: 10.1007/s43390-020-00132-2. 
25. Bagó J, Climent JM, Pérez-Grueso FJS, et al. Outcome instruments to assess scoliosis surgery. Eur Spine J 2013;22(Suppl 2):S195-S202. DOI: 10.1007/s00586-012-2352-6.

26. Koch KD, Buchanan R, Birch JG, et al. Adolescents undergoing surgery for idiopathic scoliosis: how physical and psychological characteristics relate to patient satisfaction with the cosmetic result. Spine (Phila Pa 1976) 2001;26(19):2119-2124. DOI: 10.1097/00007632200110010-00015.

27. Merola AA, Haher TR, Brkaric M, et al. A multicenter study of the outcomes of the surgical treatment of adolescent idiopathic scoliosis using the scoliosis research society (SRS) outcome instrument. Spine (Phila Pa 1976) 2002;27(18):2046-2051. DOI: 10.1097/00007632200209150-00015.

28. Scoliosis_Research_Society. [Patient Outcome Questionnaires], 2003. Available at: https://www.srs.org/professionals/online-educationand-resources/patient-outcome-questionnaires. Accessed October 9, 2018.

29. Asher M, Min Lai S, Burton D, et al. The reliability and concurrent validity of the scoliosis research society-22 patient questionnaire for idiopathic scoliosis. Spine (Phila Pa 1976) 2003;28(1):63-69. DOI: 10.1097/00007632-200301010-00015.

30. Glassman SD, Bridwell KH, Shaffrey $\mathrm{Cl}$, et al. Health-related quality of life scores underestimate the impact of major complications in lumbar degenerative scoliosis surgery. Spine Deform 2018;6(1):67-71. DOI: 10.1016/j.jspd.2017.05.003.

31. Arima $\mathrm{H}, \mathrm{Carreon} \mathrm{LY}, \mathrm{Glassman} \mathrm{SD}$, et al. Age variation in the minimum clinically important difference in SRS-22r after surgical treatment for adult spinal deformity - a single institution analysis in Japan. J Orthop Sci 2018;23(1):20-25. DOI: 10.1016/j.jos.2017.09.015.

32. Akazawa T, Minami S, Kotani T, et al. Long-term clinical outcomes of surgery for adolescent idiopathic scoliosis 21 to 41 years later. Spine (Phila Pa 1976) 2012;37(5):402-405. DOI: 10.1097/BRS.0b013e31823d2b06.

33. Akazawa T, Kotani T, Sakuma T, et al. Midlife changes of healthrelated quality of life in adolescent idiopathic scoliosis patients who underwent spinal fusion during adolescence. Eur J Orthop Surg Traumatol 2018;28(2):177-181. DOI: 10.1007/s00590-017-2027-4.

34. Rushton PR, Grevitt MP. What is the effect of surgery on the quality of life of the adolescent with adolescent idiopathic scoliosis? a review and statistical analysis of the literature. Spine 2013;38(9):786-794. DOI: 10.1097/BRS.0b013e3182837c95.

35. Chau W-W, Illescas V, Ng B. Correlation of curve flexibility analysis with patient health outcomes after scoliosis surgery using scoliosis research society-22 questionnaire. J Orthop, Traumatol Rehabilitat 2020;12(1):42-48. DOI: 10.4103/jotr.jotr_54_19.

36. Ng B, Chau W-W. Changes of shoulder balance, sagittal alignments, and curve correction in the treatment of Lenke 1 and 2 adolescent idiopathic scoliosis using a three-dimensional-based correction strategy in correlation to health-related quality of life using the scoliosis research society-22 questionnaire. J Orthoped, Traumatol Rehabilitat 2020;12(1):6-12. DOI: 10.4103/jotr.jotr_46_19.
37. Zhao J, Fan J, Chen Y, et al. A retrospective controlled clinical study of Cobb angle distribution of the main thoracic curve in adolescent idiopathic scoliosis. Medicine 2018;97(28):e11473. DOI: 10.1097/ MD. 0000000000011473 .

38. Carrasco MIB, Ruiz MCS. Perceived self-image in adolescent idiopathic scoliosis: an integrative review of the literature. Revista da Escola de Enfermagem da USP 2014;48(4):748-757. DOI: 10.1590/s0080623420140000400024.

39. Chan YL, Siu YW, Chau WW, et al. CSF motion at the cranio-cervical junction in adolescent idiopathic scoliosis - a phase contrast MR velocimetry study. International Research Society for Spinal Deformities. Vancouver, Canada 2004. p. 250.

40. Ilharreborde B, Ferrero E, Angelliaume A, et al. Selective versus hyperselective posterior fusions in Lenke 5 adolescent idiopathic scoliosis: comparison of radiological and clinical outcomes. Eur Spine J 2017;26(6):1739-1747. DOI: 10.1007/s00586-017-5070-2.

41. Chaib Y, Bachy M, Zakine $S$, et al. Postoperative perceived health status in adolescent following idiopathic scoliosis surgical treatment: results using the adapted French version of scoliosis research society outcomes questionnaire (SRS-22). Orthop Traumat Surg Res 2013;99(4):441-447. DOI: 10.1016/j.otsr.2013.03.012.

42. Sponseller PD, Flynn JM, Newton PO, et al. The association of patient characteristics and spinal curve parameters with Lenke classification types. Spine 2012;37(13):1138-1141. DOI: 10.1097/ BRS.0b013e31824054a5.

43. Mimura T, Ikegami S, Kuraishi S, et al. Residual thoracolumbar/lumbar curve is related to self-image after posterior spinal fusion for Lenke 1 and 2 curves in adolescent idiopathic scoliosis patients. J Neurosurg Pediatr 2020;26(2):211. DOI: 10.3171/2020.2.PEDS19656.

44. Bastrom TP, Bartley C, Marks MC, et al. Postoperative perfection: ceiling effects and lack of discrimination with both SRS-22 and -24 outcomes instruments in patients with adolescent idiopathic scoliosis. Spine (Phila Pa 1976) 2015;40(24):E1323-E1329. DOI: 10.1097/ BRS.0000000000001082.

45. Caronni A, Zaina F, Negrini S. Improving the measurement of healthrelated quality of life in adolescent with idiopathic scoliosis: the SRS-7, a rasch-developed short form of the SRS-22 questionnaire. Res Dev Disabil 2014;35(4):784-799. DOI: 10.1016/j.ridd.2014.01.020.

46. Sanders JO, Carreon LY, Sucato DJ, et al. Preoperative and perioperative factors effect on adolescent idiopathic scoliosis surgical outcomes. Spine (Phila Pa 1976) 2010;35(20):1867-1871. DOI: 10.1097/ BRS.0b013e3181efa6f5.

47. Wang L, Wang YP, Yu B, et al. Relation between self-image score of SRS-22 with deformity measures in female adolescent idiopathic scoliosis patients. Orthop Traumatol Surg Res 2014;100(7):797-801. DOI: 10.1016/j.otsr.2014.06.014.

48. Aghdasi B, Bachmann KR, Clark D, et al. Patient-reported outcomes following surgical intervention for adolescent idiopathic scoliosis: a systematic review and meta-analysis. Clin Spine Surg 2020;33(1):2434. DOI: 10.1097/BSD.0000000000000822. 